\title{
RESPONSE OF NILE TILAPIA Oreochromis niloticus TO FED DIETS SUPPLEMENTED WITH GRADED LEVELS OF BLACK SEED Nigella sativa L. STORED UNDER TROPICAL CONDITIONS \\ Qatnan, K. A. and M. A. Al-Owafeir* \\ * Dept. of Animal and Fish Production, \\ College of Agricultural and Food Sciences,King Faisal University, \\ PO Box 55135, Hofuf, 31982, Saudi Arabia, malowafeir@kfu.edu.sa
}

\section{استجابة أسماكك البلطي النيلي Oreochromis niloticus للأعلاف المدعمة

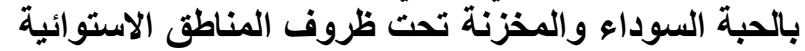

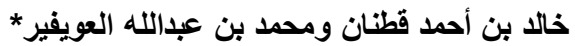 * قسم الإتتاج الحيواني والسمكي، كلية العلوم الزيراعية والأغذية، جامعة الملك فيصل،

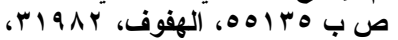 malowafeir@kfu.edu.sa المملكة العربية السعودية}

$$
\text { الملخص }
$$

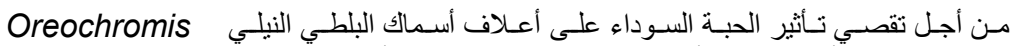

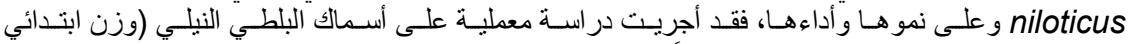

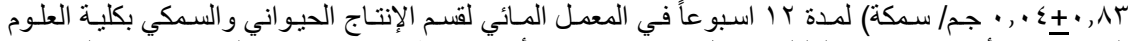

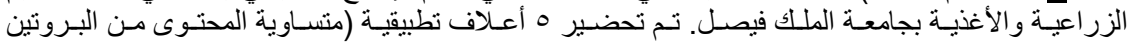

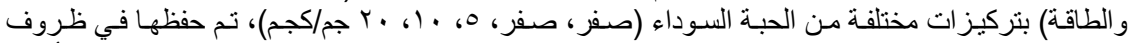

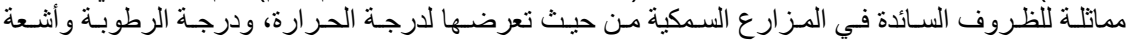

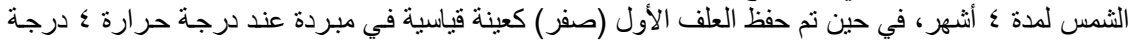

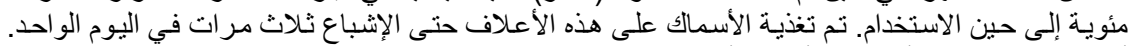

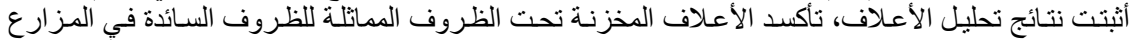

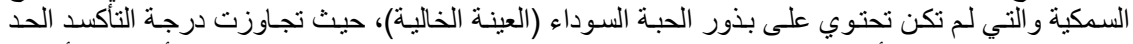

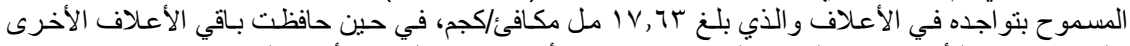

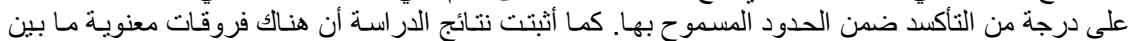

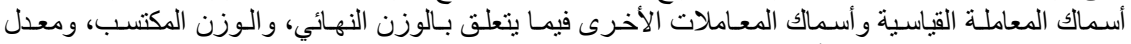

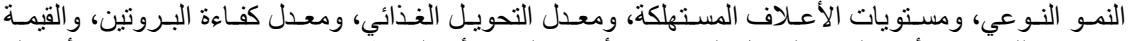

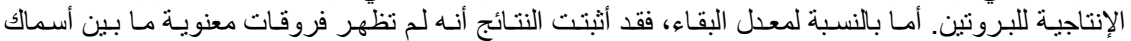

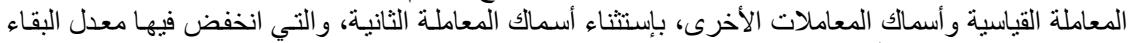

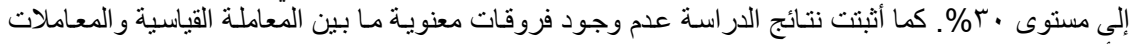

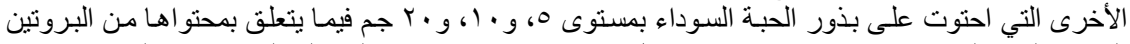

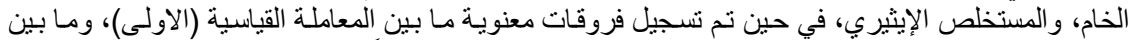

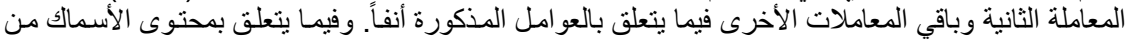

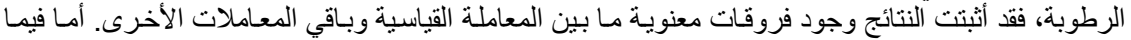

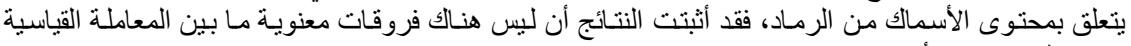

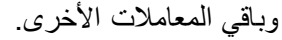

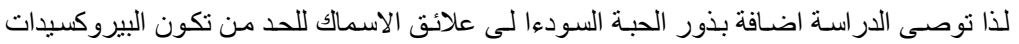

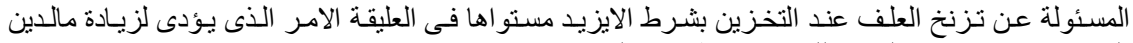
الصابونين شهية الاسماك وكذلك تحسين تُفين ظروف التخزين. 


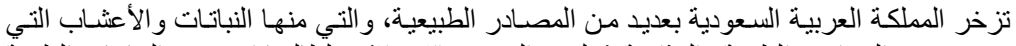

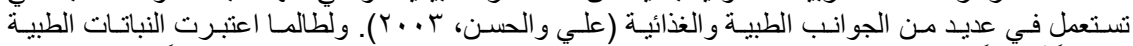

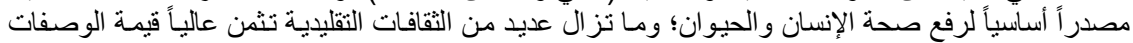

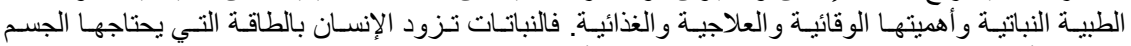

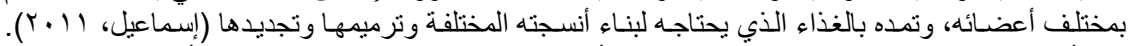

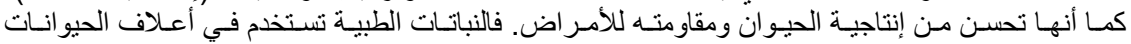

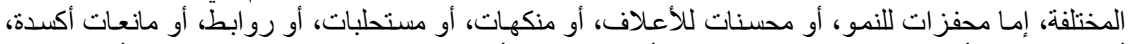

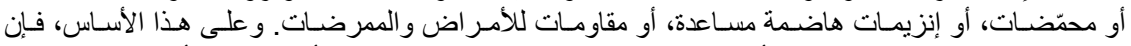

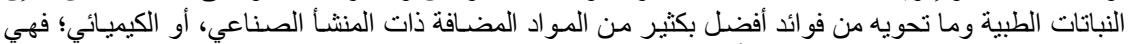
(Schrägle and Muller, 1990; نخلوي، Pradeep et al., 1991; Abd-Rabo et al., 1992; El-Emary, 1993; Hanafy, 1989; Abd El-Maksoud et al., 1999; Abd El-Latif et al., 2004)

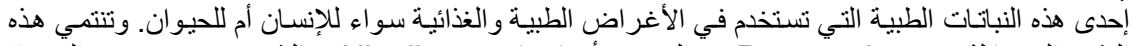

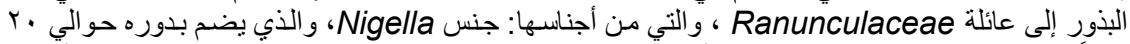

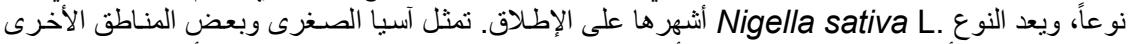

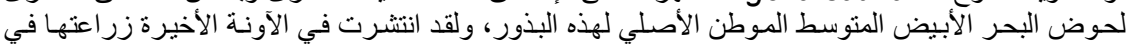

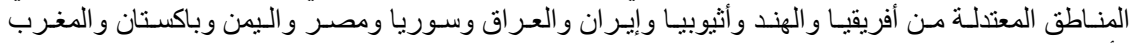

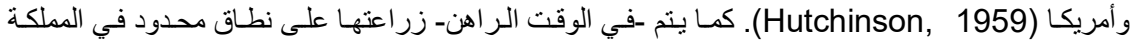

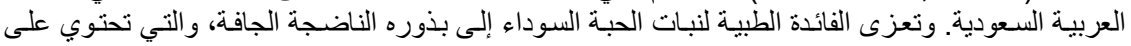

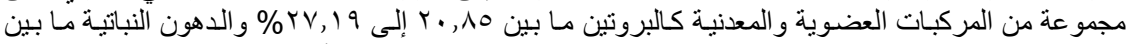

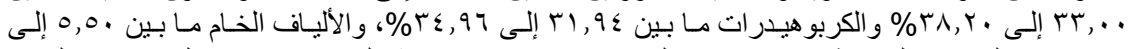

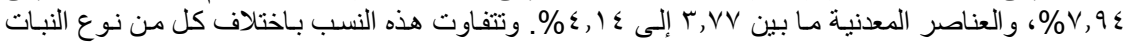

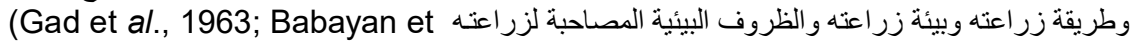
al., 1978; Greenish, 1880; Rathee et al., 1982; Al-Jassir, 1992; Abdel-Aal and (Attia, 1993)

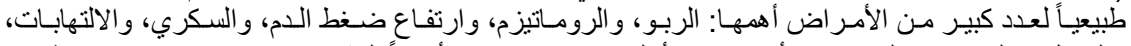

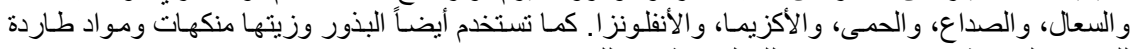

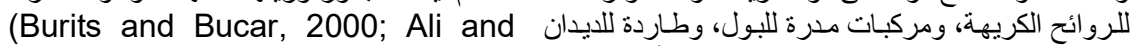

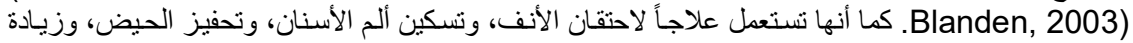

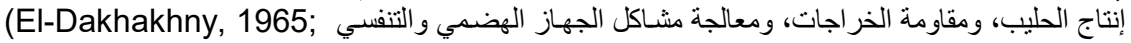
(Swamy and بالإضـافة إلى استخدامها مسادة مضسادة للفطريسات، ومضـادة للبكتيريا. Goreja, 2003) كما استخدمت مـادة مضافة إلى العلائق السمكية، فقد تم .Tan, 2001; Al-Naggar et al., 2003) (Abd El-Maksoud et Oreochromis niloticus إضافتها إلى علائق كل من أسماك البلطي النيلي al., 1998a,b; Malik and Mohsen, 2006; Soliman et al., 2000) Cyprinus carpio

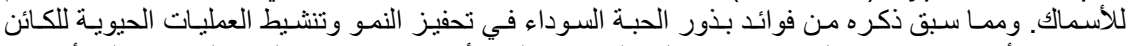

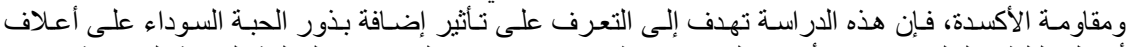

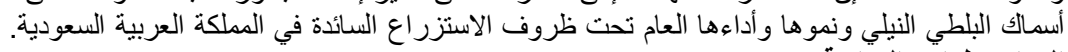

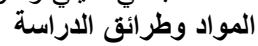

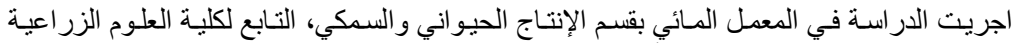

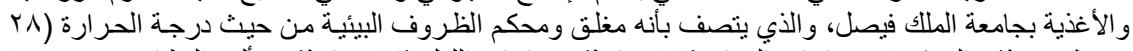

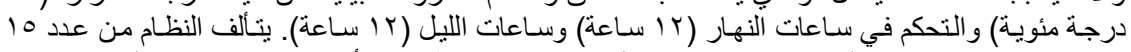

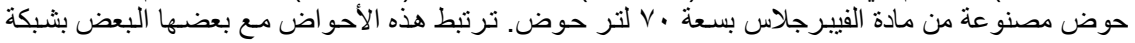

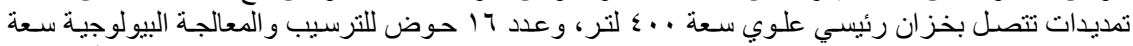

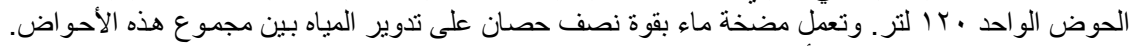

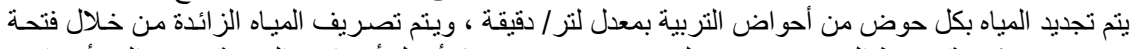

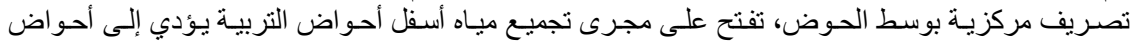




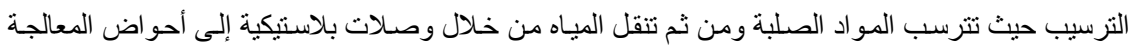

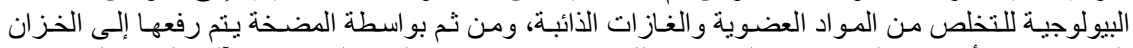

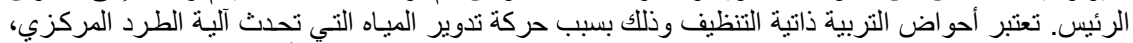

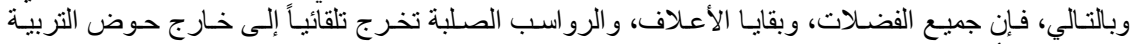

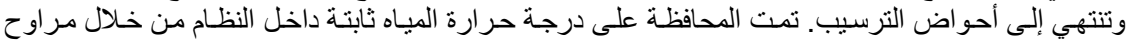

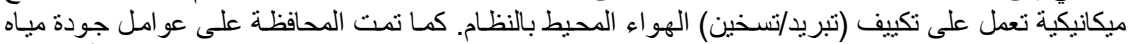

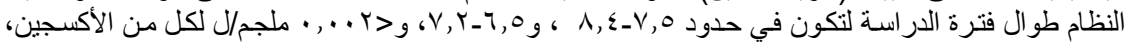

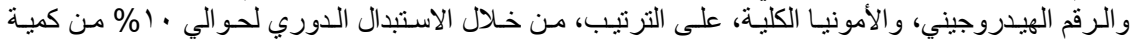

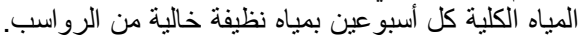

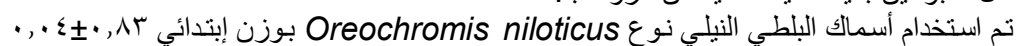

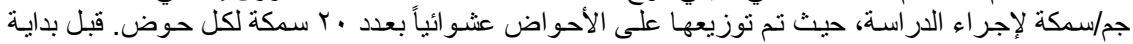

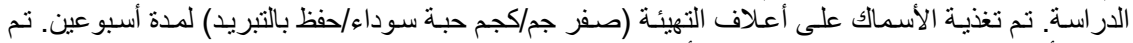

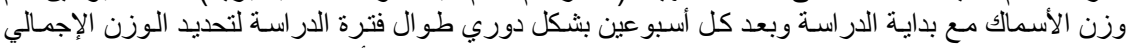

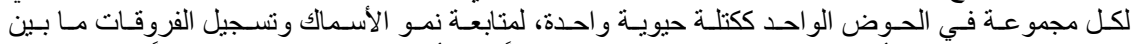

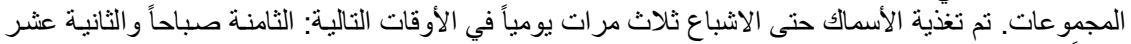

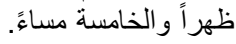

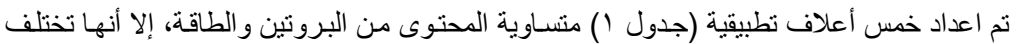

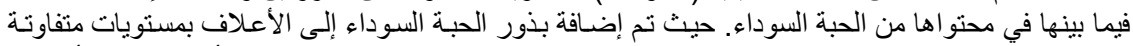

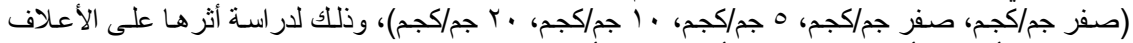

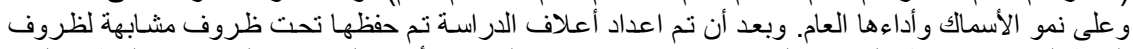

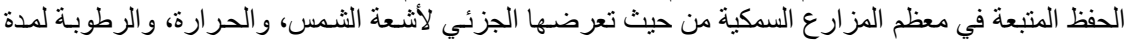

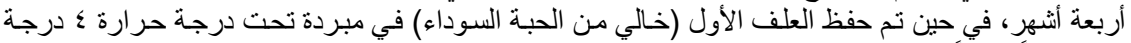
مئوية علفاً قياسباً.

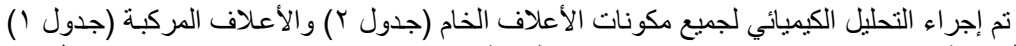

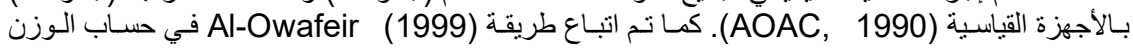

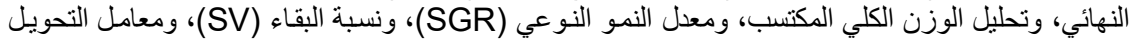

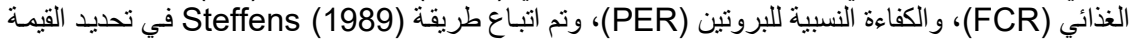

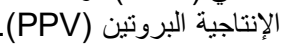

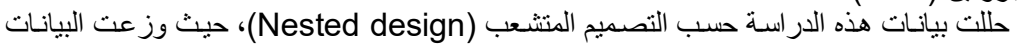

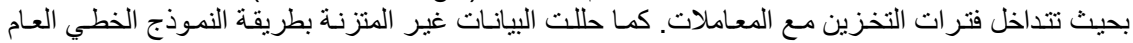

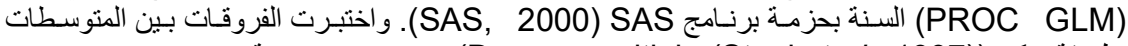
بطريقة دنكن (Duncan multiple (Steel et al., 1997) عند مستوى معنوية 0 ., ".

\section{النتائج والمناقشة}

رقم البيروكسيد للأعلاف المخزنة تحت ظروف التخزين المختلفة

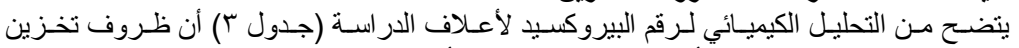

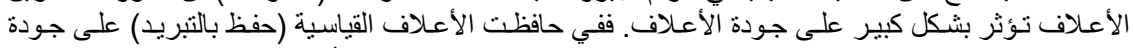

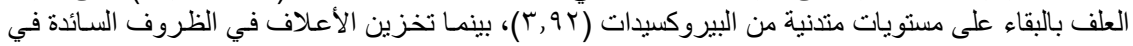

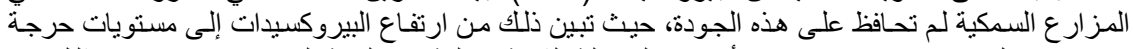
(IV,T T )

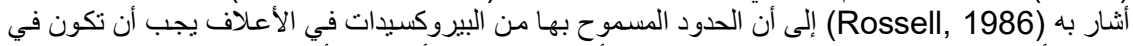

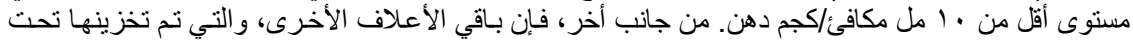

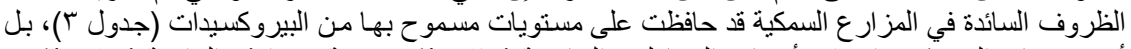

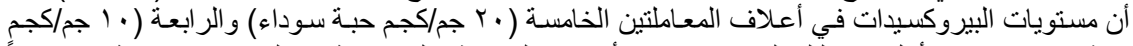

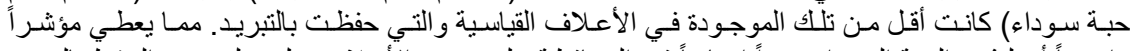
و واضحاً أن لبذور الحبة السوداء دوراً إيجابياً في المحافظة على جودة الأعلى الأعلاف بعلو على بلى دور الحفظٍ بالتبريد 


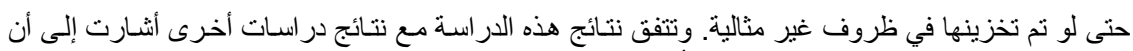

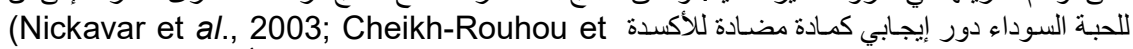

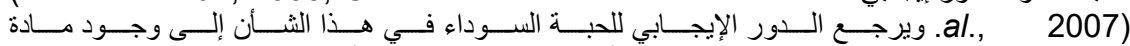

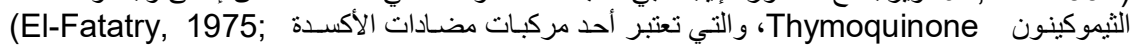
(Meral et al., 2001; El-Dakhakhny., 2002; Mahmoud et al., 2002)

كبح تكون الجذور الحرة وتثيط نأكسد الدهون (Burits and Bucar, 2000).

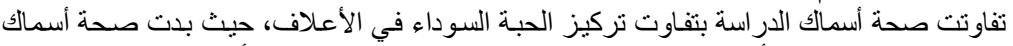

صحة الأسماك ومظهر الجذها العام

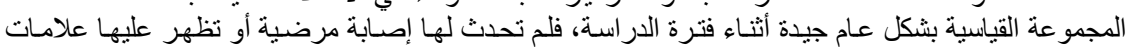

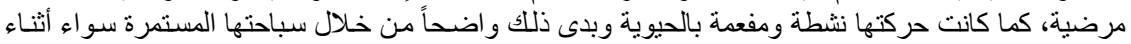

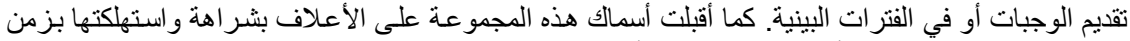

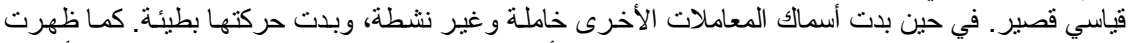

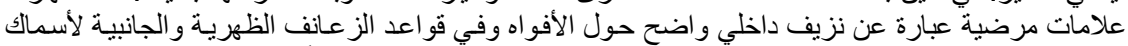

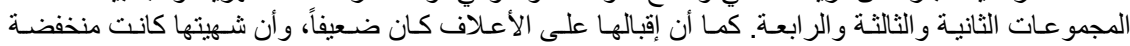

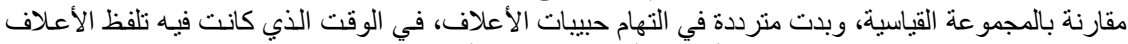

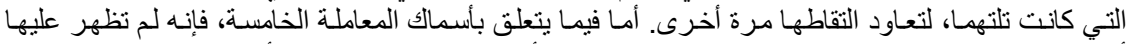

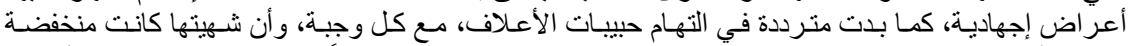

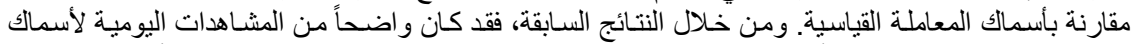

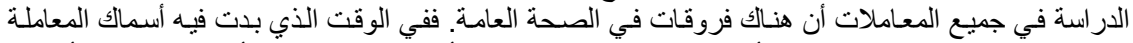

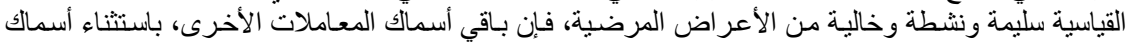

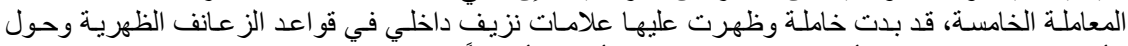

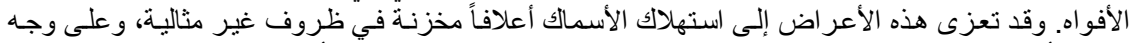

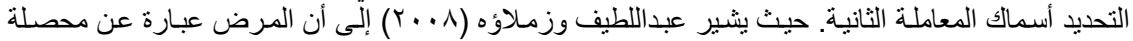

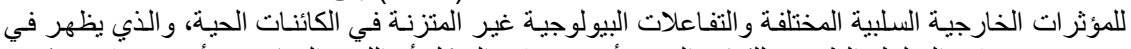

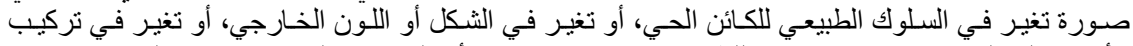

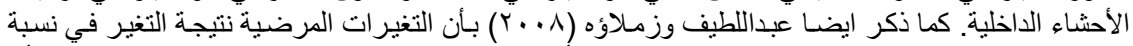

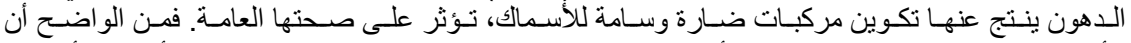

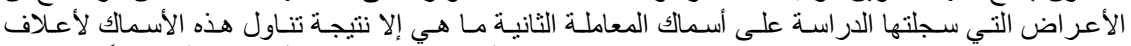

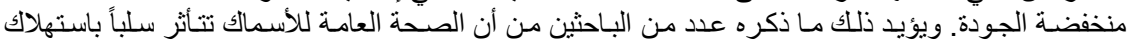

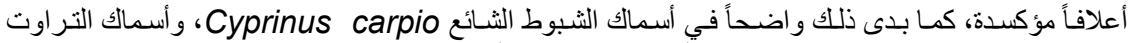
القزحية Oncorhynchus mykiss، وأسماك السلمون الأحمر Oncorhynchus (Tacon, 1990)

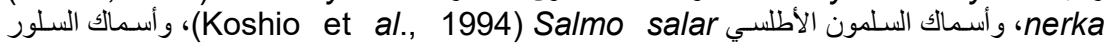
الأفريقي Clarias gariepinus (Baker and Davies, 1997)، وأسـماك البلطـي الهجـين (Huang and Huang, 2004) Oreochromis Niloticus × O. Aureus الأطلسي Martins et al., 2007) Hippoglossus Hippoglossus) أمسا بالنسبة للأعر اض التي التي

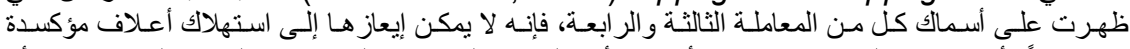

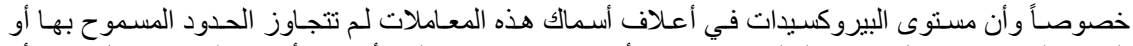

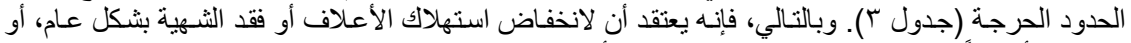

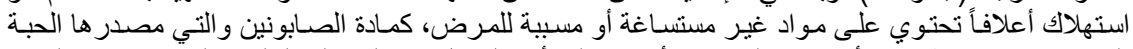

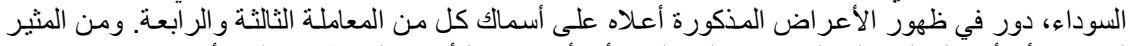

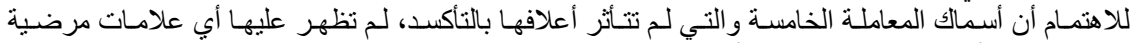

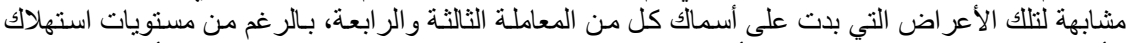

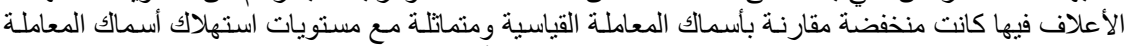

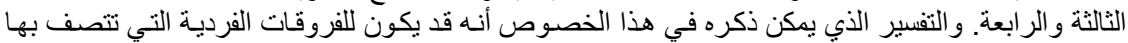

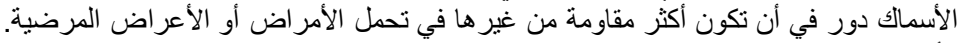

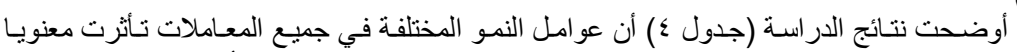

الأداء العام

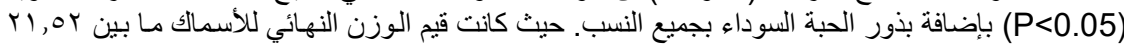




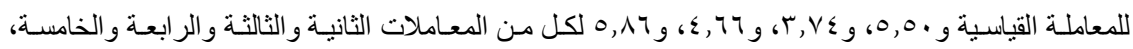

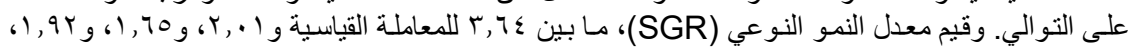

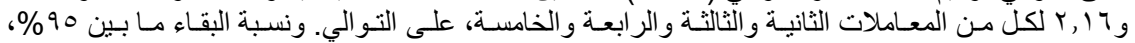

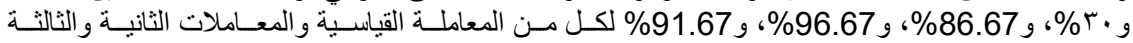

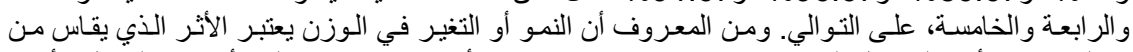

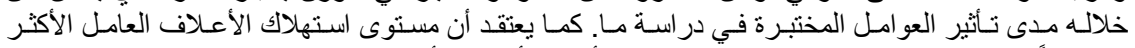

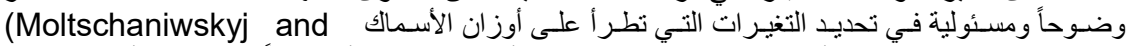

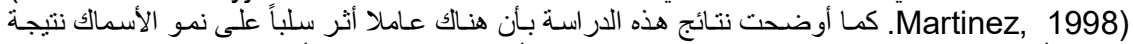

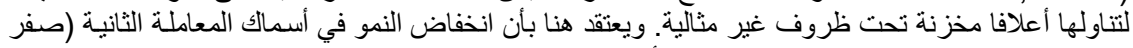

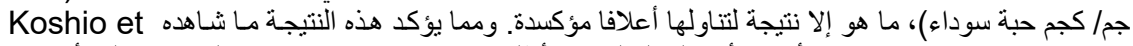

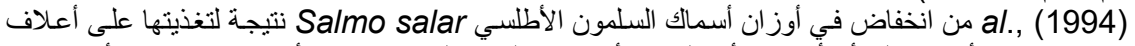

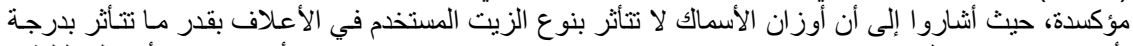

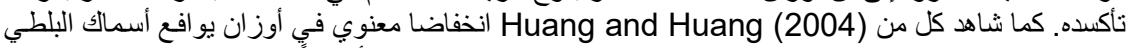

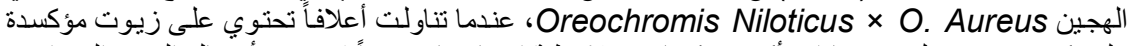

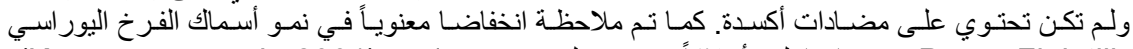
ومن Perca Fluiatilis

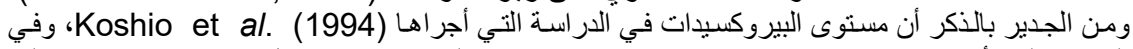

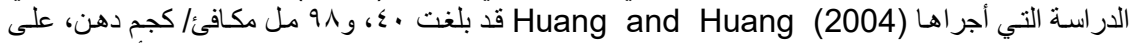

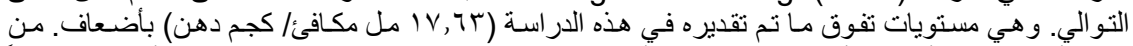

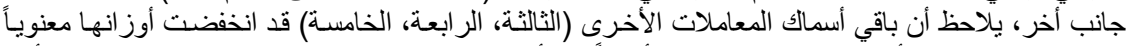

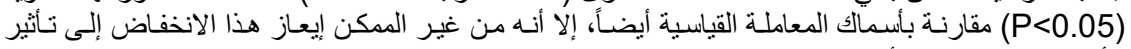

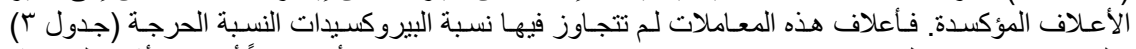

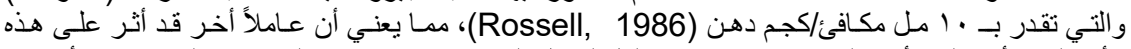

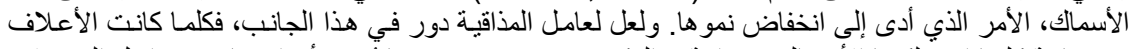

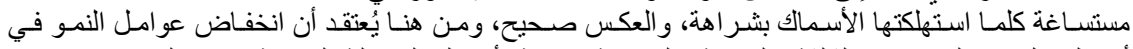

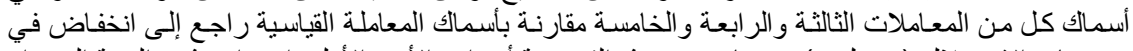

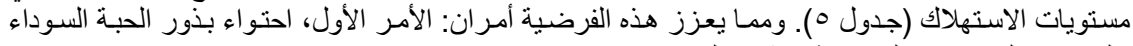

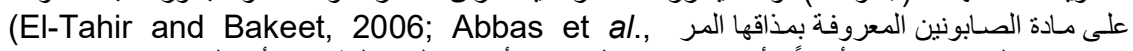

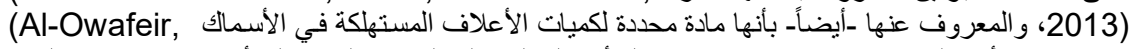

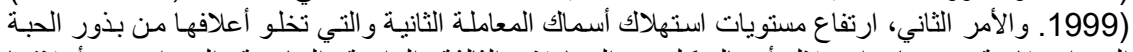

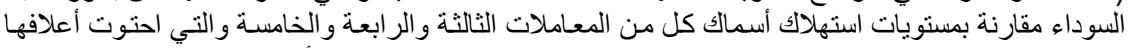

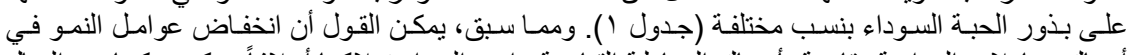

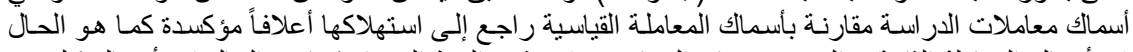

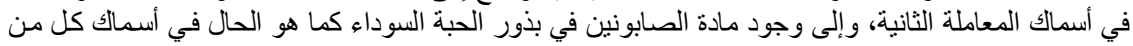

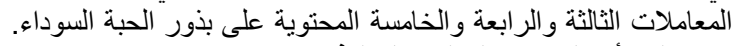

استهلاك الأعلاف ومعامل التحويل الغذائي (FCR)

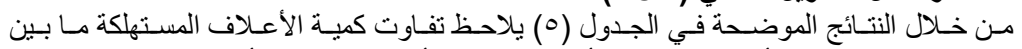

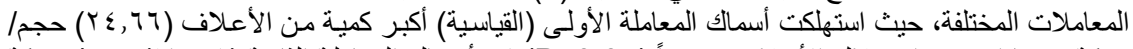

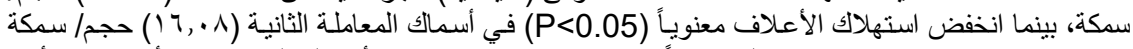

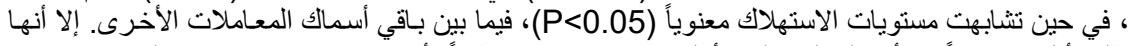

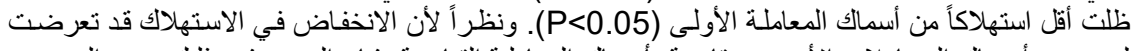

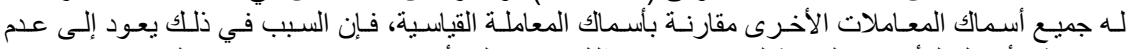

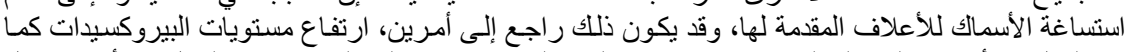

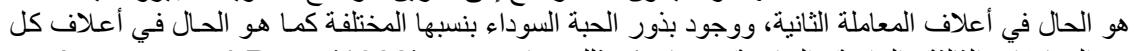

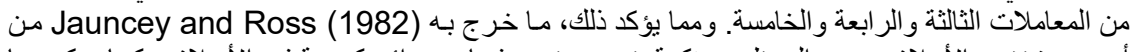

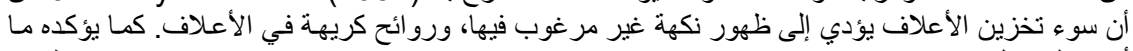

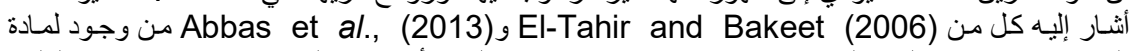
الصابونين في بذور الحبة السوداء. ومما هو معروف عن هذه المسادة أنها مرة المذاق وتؤثر في مذاقية العلف 
(Birk and Peri, 1980)، الأمر الذي أدى إلى انخفاض استهلاك الأعلاف في المعاملات المشـار إليها

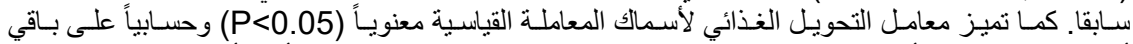

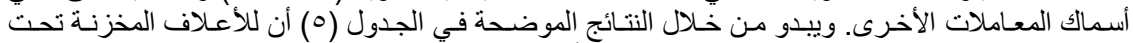

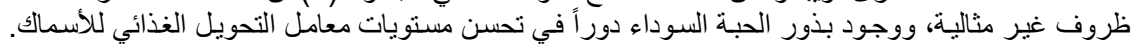

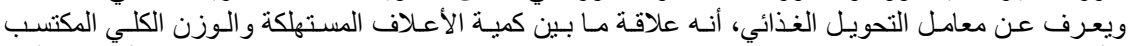

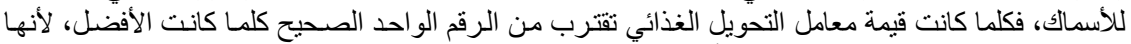

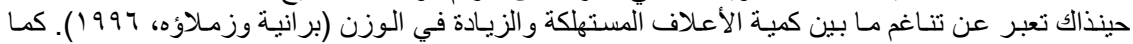

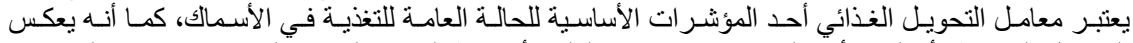

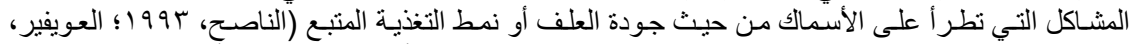

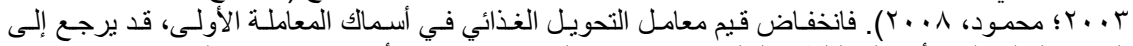

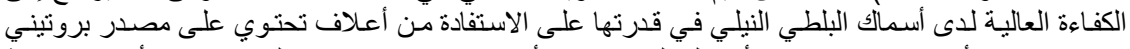

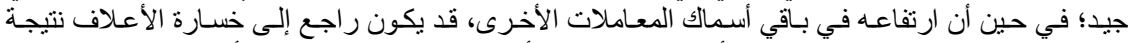

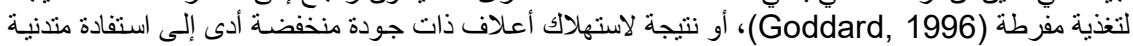

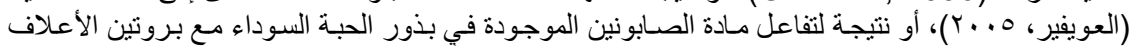

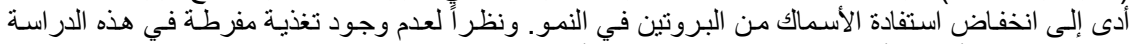

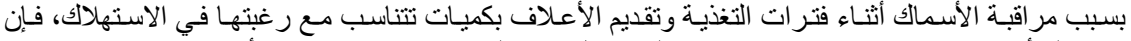

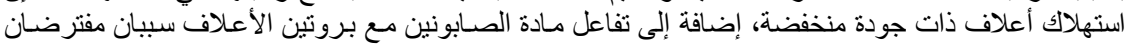

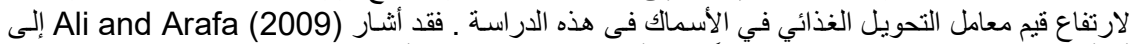

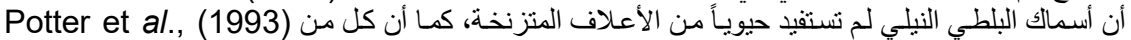

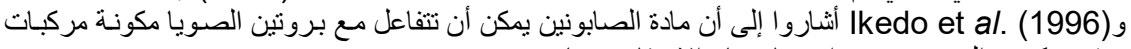
المعدة وكبيرة الحجم يصعب امتصاصها و الاستفادة منها. الكفاءة الغذائية للأعلاف الكفاء

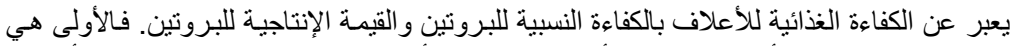

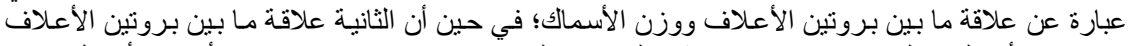

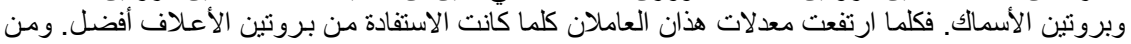

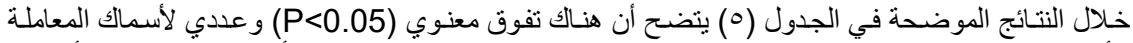

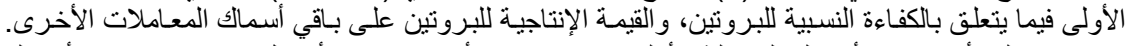

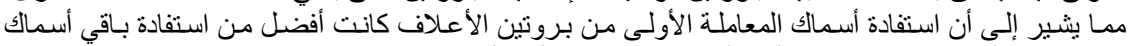

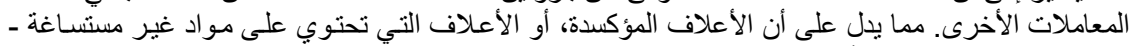

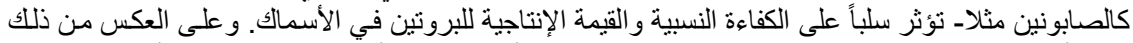

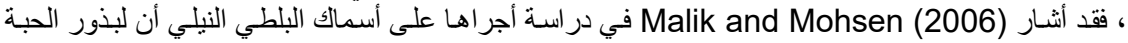

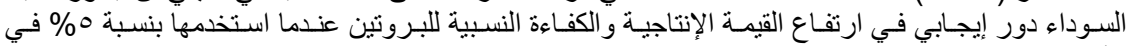

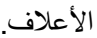

التحليل الكيميائي لأسماك الاراسة

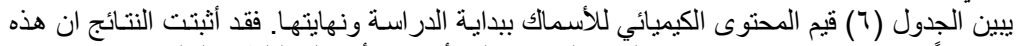

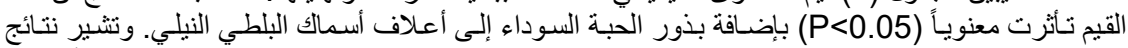

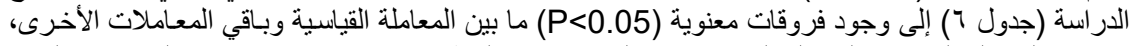

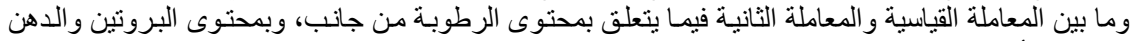

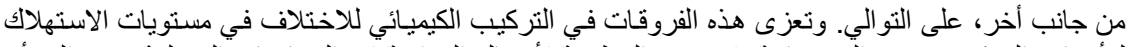

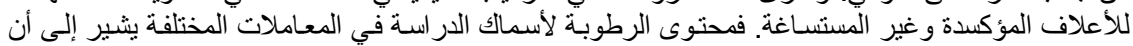

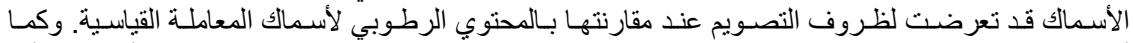

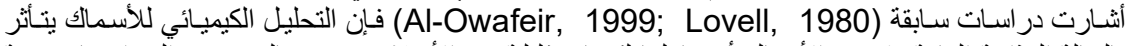

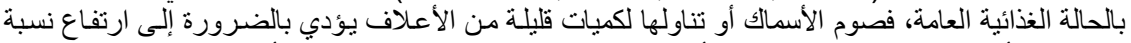

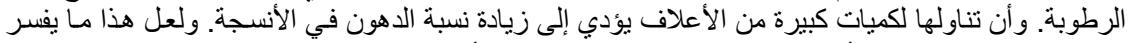

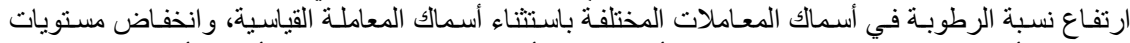

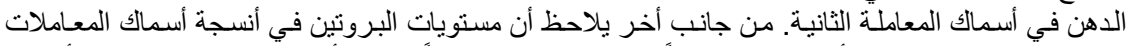

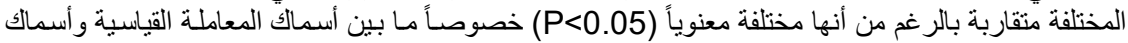

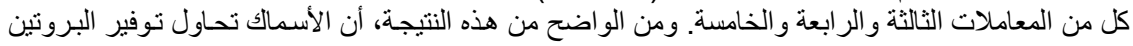


أثنـاء ظروف التصـويم، أو عند انخفاض استهلاك الغذاء. وممـا يتو افق مـع هذه النتيجـة، مـا أثـار إليـه هـانج

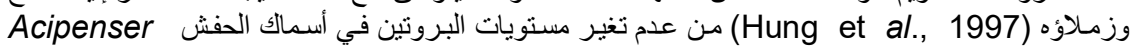
Transmontanus

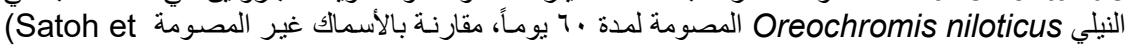

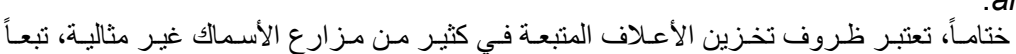
.al., 1984)

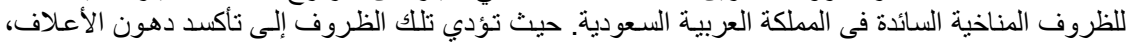

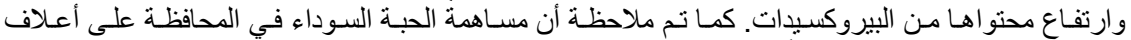

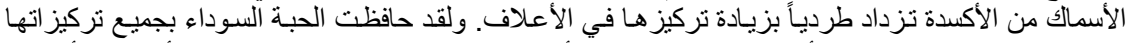

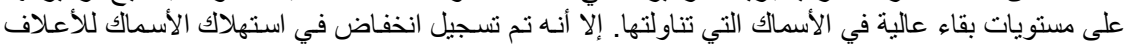

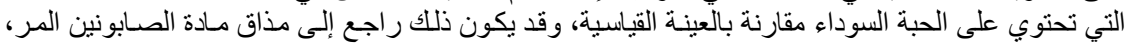

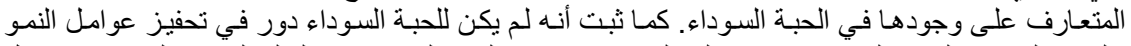

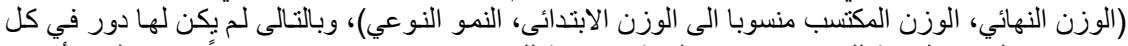

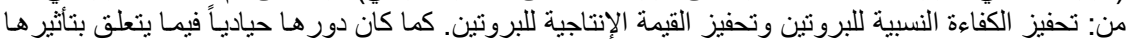
على التركيب الكيميائي للحوم الأسماك. لاكت 


\begin{tabular}{|c|c|c|c|c|c|}
\hline \multicolumn{6}{|c|}{ جدول (1) تركيب الأعلاف المستخدمة في الدراسة (جم/كجم من الوزن الرطب) } \\
\hline علف & علف؛ & علف & علف & علف & المكونات \\
\hline 074,1 & 074,1 & OT, & $07 \pi, 1$ & 074,1 & فول الصويا \\
\hline 111,9 & 111,9 & 111,9 & $1 \wedge 7,9$ & $1 \wedge 7,9$ & ذرة صفراء \\
\hline 00 & 70 & $V \cdot$ & $V$. & $V$. & نخالة القمح \\
\hline$r$. & $r$. & r. & r. & $r$. & فيتامينات \\
\hline $1 \ldots$ & $1 \ldots$ & $1 \cdots$ & $1 \ldots$ & $1 \ldots$ & زيت الذره \\
\hline$\varepsilon$ & $\varepsilon$ & $\varepsilon \cdot$ & $\varepsilon$ & $\varepsilon$ & معادن \\
\hline r. & $r$. & $r \cdot$ & r. & $r \cdot$ & مو اد رابطة \\
\hline$r \cdot$ & $1 \cdot$ & 0 & صفر & صفر & الحبة السوداء \\
\hline $1 \ldots$ & $1 \cdots$ & $1 \cdots$ & $\ldots$ & $1 \ldots$ & المجموع \\
\hline \multicolumn{6}{|c|}{ المحتوى الكيميائي لأعلاف الدراسة (جم/كجم من الوزن الجاف) } \\
\hline علف & علف؛ & علفr & علف & علف' & \\
\hline$M T, V$ & $r \cdot 9, \wedge$ & $r \cdot \Lambda, r$ & $r q \leq, V$ & $r \cdot r, r$ & البروتين الخام \\
\hline IYO,. & ITr,. & $11 \%, \cdot$ & $111, \cdot$ & $11 \varepsilon$, & المستخلص الإيثيري \\
\hline$r \varepsilon$, & $0 \leqslant, 1$ & $\leqslant 0,7$ & $\varepsilon \vee, V$ & $r \cdot, r$ & الألياف الخام \\
\hline$\wedge \wedge, 1$ & $\wedge 9, \Sigma$ & $9 \wedge, \vee$ & $90, \cdot$ & $9 \varepsilon$, & الرماد \\
\hline$\varepsilon r q, r$ & $\varepsilon r T, V$ & $\varepsilon r \leqslant, 0$ & $\leqslant \varepsilon \leqslant, 7$ & $\sum 0 \wedge, 1$ & المستخلص الخالي من النيتروجين \\
\hline$r, 90$ & $r, q 1$ & $r, \Lambda$. & $r, V V$ & $r, v q$ & إجمالي الطاقة كالوري/جم \\
\hline \multicolumn{6}{|c|}{ 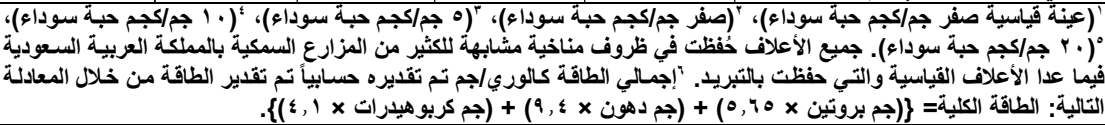 } \\
\hline
\end{tabular}

\begin{tabular}{|c|c|c|c|c|c|c|}
\hline \multicolumn{7}{|c|}{ جدول (ץ) التحليل الكيميائي للمواد الخام المستخدمة في الدراسة (وزن رطب) } \\
\hline إجمالي الطاقة كيلو & المن النيتروجين الخالي & 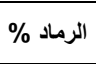 & الألياف الخام \% & الإيشيري \% المستخل & البروتين الخام & |المادة الخام \\
\hline$\varepsilon, Y_{O}$ & $r \neg, I V$ & 7,19 & $\varepsilon, 79$ & $r, 07$ & 01,91 & فول الصويا \\
\hline$r, V V$ & $v r, Y q$ & $1, Y Y$ & $r, \varepsilon \varepsilon$ & $r, \leqslant q$ & $V, T)$ & الذرة الصفر اء \\
\hline$r, \leqslant q$ & 00,00 & $\varepsilon, \varepsilon Y$ & $11, \cdot r$ & r,OY & IV,YY & نخالة القمح \\
\hline $0, \varepsilon V$ & $1 Y, 07$ & $\varepsilon, \varepsilon\rceil$ & $1 Y, \leqslant 7$ & $r v, V \varepsilon$ & $r \leqslant, 70$ & الحبة السوداء \\
\hline \multicolumn{7}{|c|}{ 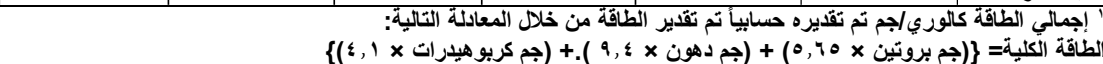 } \\
\hline
\end{tabular}

\begin{tabular}{|c|c|c|c|c|c|}
\hline \multicolumn{6}{|c|}{ جلول (r) التحليل الكيميائي لرقم البيروكسيد في أعلاف الدراسة } \\
\hline \multicolumn{3}{|c|}{ رقم البيروكسبد (مل مكافئ/كجم، دهن) } & \multicolumn{3}{|c|}{ أعلاف المعاملات } \\
\hline \multicolumn{3}{|c|}{$r, q r$} & \multicolumn{3}{|c|}{ الأولى (صفر جم/كجم حبة سوداء عبنة قياسية، حفظ بالتبريد) } \\
\hline \multicolumn{3}{|c|}{ IV,TT } & \multicolumn{3}{|c|}{ الثانية (صفر جم/كجم حبة سوداء) } \\
\hline \multicolumn{3}{|c|}{$\varepsilon, \vee \cdot$} & \multicolumn{3}{|c|}{ الثالثة (0 جم/كجم حبة سوداء) } \\
\hline \multicolumn{3}{|c|}{$r, r q$} & \multicolumn{3}{|c|}{ الرابعة (· ( جم/كجم حبة سوداء) } \\
\hline \multicolumn{3}{|c|}{1,91} & \multicolumn{3}{|c|}{ الخامسة (•r جم/كجم حبة سوداء) } \\
\hline \multicolumn{6}{|c|}{ جدول (ء) أداء النمو ونسب البقاء في أسماك الدراسة } \\
\hline نسبة البقاء (\%) & معدل النمو النوعي & الوزن المكتسب (\%) & 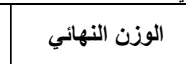 & الوزن الابتدائي & المعاملات \\
\hline$\omega_{0, \varepsilon \wedge \pm 90}$ & $\omega, 10 \pm r, 1 \varepsilon$ & $ص q \cdot, \cdot r \pm Y \circ r v, \varepsilon \varepsilon$ & $\omega \cdot, V \vee \pm Y 1,0 Y$ & ${ }^{\omega} 0.02 \pm 0.82$ & الأولى' \\
\hline $20, \Sigma \wedge \pm \Gamma$ & $\varepsilon \tau_{\cdot}, 10 \pm r, \cdot 1$ & $2 q \cdot, \cdot r \pm 00 \cdot, 1 \wedge$ & $\tau_{\cdot}, V \vee \pm 0,0$ & ${ }^{\infty} 0.02 \pm 0.84$ & الثانيةّم \\
\hline 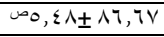 & $\varepsilon_{\cdot, 10 \pm 1,70}$ & $\tau q \cdot, \cdot r \pm r \leqslant \lambda, \vee$. & $\tau_{\cdot}, V V_{ \pm} r, V \varepsilon$ & ${ }^{\omega} 0.02 \pm 0.83$ & الثالثة \\
\hline$\omega_{0, \Sigma \wedge \pm 97,7 V}$ & $\varepsilon \tau \cdot, 10 \pm 1,9 r$ & $\tau q \cdot, \cdot r \pm \sum T V, Y \cdot$ & $\tau \cdot, V V_{ \pm} \varepsilon, 7 \uparrow$ & $\omega^{\infty} 0.02 \pm 0.82$ & الرابعة' \\
\hline 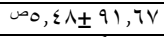 & $2 \cdot, 10 \pm Y, 17$ & $\tau q \cdot, \cdot r \pm \tau \cdot r, r$ & $\tau \cdot, \vee \vee \pm 0, \wedge \tau$ & ${ }^{-0.02 \pm 0.83}$ & الخامسةٌ \\
\hline
\end{tabular}

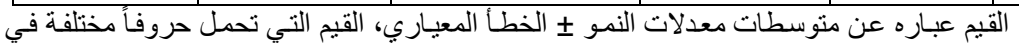

أعدة: الجداول تعتبر مختلفة معنويا (P<0.05). 


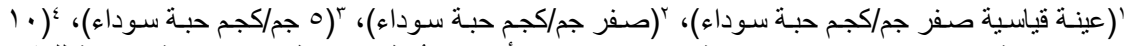

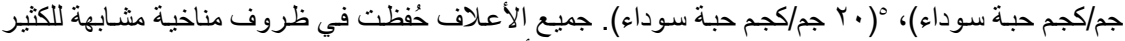
من المزارع السمكية بالمملكة العربية السعودية فيما عداء الأعلاف القياسية والتية والتي حفظت بالتبريد.

\begin{tabular}{|c|c|c|c|c|}
\hline القيمة الإنتاجية & الكفاءة النسبية & معامل التحويل & كمية العلفة & \\
\hline$\omega r, V r_{ \pm} \leqslant V, q r$ & ص. , $r \leq \pm r, q \leq$ & r, & $\begin{array}{c} \pm 23.23 \\
-1.46\end{array}$ & الأولى' \\
\hline$\tau T, r \leq \pm Y \cdot, \Lambda T$ & $\tau_{\cdot}, r \leq \pm \cdot, q$. & $\tau_{\cdot}, 0 r \pm r, V r$ & $\ulcorner 1.46 \pm 15.05$ & 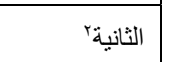 \\
\hline$\varepsilon \tau Y, V T \pm I \leqslant, 0$. & $\tau_{\cdot}, r \leq \pm 1, \cdot 0$ & $\tau \cdot, 0 Y \pm T, 17$ & $\varepsilon 1.46 \pm 8.94$ & 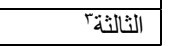 \\
\hline$\tau Y, V \Psi \pm 1 \wedge, \cdot r$ & $\tau \cdot, Y \leq \pm 1, r V$ & $\varepsilon_{\cdot}, O Y \pm Y, r Y$ & $\varepsilon 1.46 \pm 9.04$ & 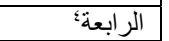 \\
\hline$\omega \tau r, V r \pm Y r, q r$ & $\varepsilon_{\cdot}, Y \leq \pm 1, \lambda 1$ & $\omega_{\cdot, 0 r \pm 1, \lambda l}$ & $\varepsilon 1.46 \pm 8.98$ & الخامسةة \\
\hline \multicolumn{5}{|c|}{ 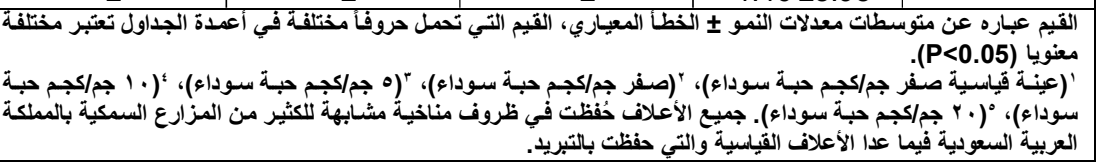 } \\
\hline \multicolumn{5}{|c|}{ جدول (T) التحليل الكيميائي للأسماك في بداية ونهاية الدراسة (وزن رطب) } \\
\hline $\begin{array}{l}\text { الرماد) } \\
\text { (\%) }\end{array}$ & المستخلص الإيشيري & $\begin{array}{c}\text { البروتين الخام } \\
\text { (\%) }\end{array}$ & $\begin{array}{c}\text { الزطوبة } \\
\text { (\%) }\end{array}$ & المعاملات \\
\hline 3.15 & 5.77 & 14.66 & $V T, \varepsilon Y$ & الدر اسـة الك بدايــــة \\
\hline$\omega, Y \cdot \pm \varepsilon, \cdot r$ & $\omega_{\cdot}, \Sigma \Sigma \pm \Lambda, Y Y$ & 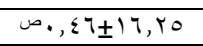 & 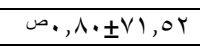 & الأولى' \\
\hline$ص, r\rceil \pm \varepsilon, 11$ & $\tau_{0}, 07 \pm r, T V$ & $\tau \omega, 0 q \pm 1 \leqslant, V V$ & $\tau_{\cdot}, 9 \vee \pm V V, I V$ & الثانية \\
\hline 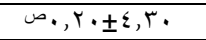 & $\omega_{\bullet}, \leq \varepsilon \pm \nearrow, V \vee$ & $\tau_{\cdot}, \leq 7 \pm 1 r, 99$ & $\tau \cdot, \Lambda \cdot \pm V \leqslant, 90$ & الثالثةr \\
\hline$\omega \cdot, r \cdot \pm r, v q$ & $\omega_{.}, \varepsilon \Sigma \pm \vee, \Lambda l$ & $\tau_{\cdot}, \leq \neg \pm 1 T, \leqslant Y$ & $\tau \cdot, \wedge \cdot \pm \vee \leqslant, q \wedge$ & الر ابعة؛ \\
\hline 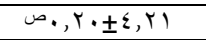 & $\omega_{\cdot}, \varepsilon \leqslant \pm V, r$. & $\tau \cdot, \leqslant\rceil \pm \mid r, r q$ & $\tau \cdot, \Lambda \cdot \pm \vee \bullet, Y I$ & الخامسة. \\
\hline
\end{tabular}

. $(\mathrm{P}<0.05)$

\section{المراجع العربية البية}

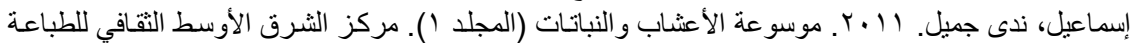

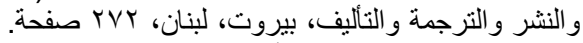

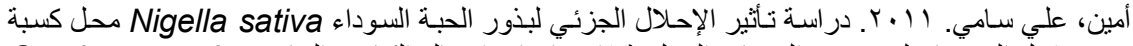

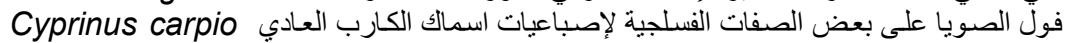

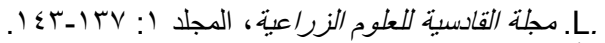

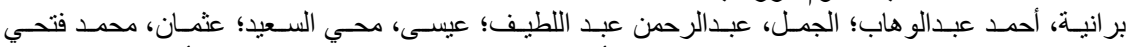

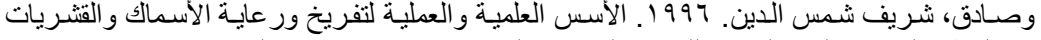

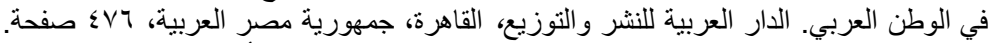

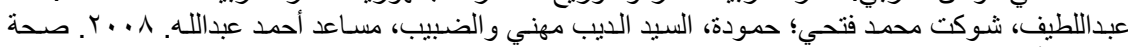

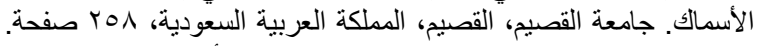

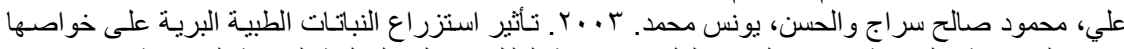

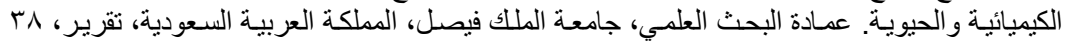
صفحة.

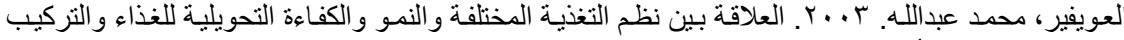

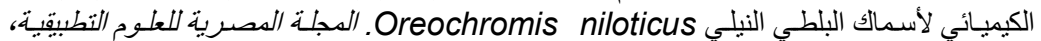

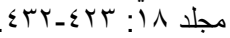




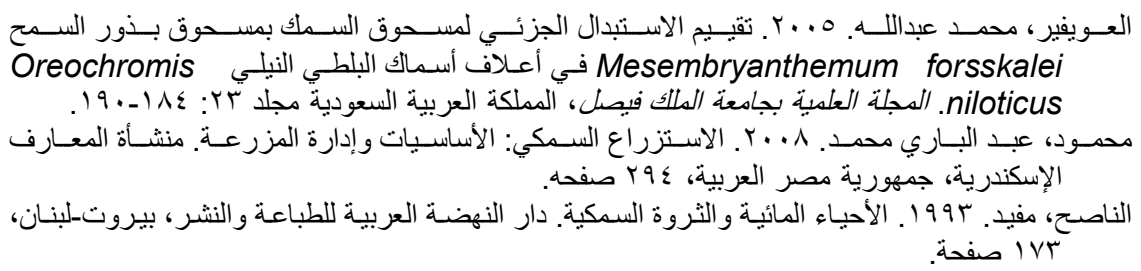

Abbas, M., Shahid, M., Iqbal, M., Anjum, F., Sharif, S., Ahmed, S. and Pirzada, T. 2013. Antitermitic Activity and Phytochemical Analysis of fifteen medicinal plant seeds. Journal of medicinal Plant Research, 7: 1608-1617.

Abd El-Latif, S. A., El-Yamany, A. T. and Edaly, A. F. 2004. Evaluation of Using Different Levels and Sources of Medicinal Herbs in Growing Japanese Quail Diets. Egyptian Journal of Nutrition and Feeds, 7: 69-81.

Abd El-Maksoud, A. M. S., Aboul-Fotouh, G. E., Allam, S. M. and Abou Zied, R. M. 1999. Effect of Marjoram Leaves (Majorana hortensis L.) as a Feed Additive on the Performance of Nile Tilapia (Oreochromis niloticus) Fingerlings. Egyptian Journal of Nutrition and Feeds, 2: 39-47.

Abd El-Maksoud, A. M. S., Aboul-Fotouh, G. E., Allam, S. M. and Abou Zied, R. M. 1998a. Evaluation of Nigella Seeds (Nigella sativa L.) as a Feed Additive in Diets of Nile Tilapia (Oreochromis niloticus). Fayoum Journal of Agricultural of Research \& Development, 12: 170-181.

Abd El-Maksoud, A. M. S., Aboul-Fotouh, G. E., Allam, S. M. and Abou Zied, R. M. 1998b. The Effect of Feeding Diets Containing Chamomile Flowers on Growth, Survival and Feed Utilization of Nile Tilapia (Oreochromis niloticus) Fingerlings. Fayoum Journal of Agricultural of Research \& Development, 12: 181-192.

Abdel-Aal, E. S. M. and Attia, R. S. 1993. Characterization of Black Cumin (Nigella sativa L.) Seeds. 1-Chemical Composition and Lipids. Journal of Alexandria Science Exchange, 14: 467-482.

Abd-Rabo, F. H., Attia, M. H., Soliman, A. Z. and Ali, H. M. 1992. Effect of Garlic Oil and Some of its Sulfur Containing Constituents on Serum and Milk Cholesterol and the Efficiency of Feed Utilization in Rabbits. Egyptian journal of applied science, 7: 846-856.

Ali, A. T. and Arafa, M. M. 2009. Field Studies of Cultured Tilapia Niloticus Fish Fed on Rancid Ration. Egyptian Journal of Comparative Pathology and Clinical Pathology, 22: 151-163.

Ali, B. H. and Blunden, G. 2003. Pharmacological and Toxicological Properties of Nigella sativa. Phytotherapy Research, 17: 299-305.

Al-Jassir, M. S. 1992. Chemical Composition and Microflora of Black Cumin, Nigella sativa L. Seeds Growing in Saudi Arabia. Journal of Food chemistry, 45: 239-242.

Al-Naggar, T. B., Gomez-Serranillos, M. P., Carreto, M. E. and Villar, A. M. 2003. Neuropharmacological Activity of Nigella sativa L. Extracts. Journal of Ethnopharmacology, 88: 63-68.Egypt.

Al-Owafeir, M. 1999. The Effects of Dietary Saponin and Tannin on Growth Performance and Digestion in Oreochromis niloticus and Clarias 
gariepinus, PhD. Thesis, Institute of Aquaculture, University of Stirling, Scotland.

AOAC 1990. Official Methods of Analysis, 15th ed. Association of Official Analytical Chemists, Arlington,Virginia.

Babayan, V. K., Koottungal, D. and Halaby, G. A. 1978. Proximate Analysis, Fatty acid and Amino acid Composition of Nigella sativa L. Seeds. Journal of Food Science, 43: 1314-1315.

Baker, R. T. M. and Davies, S. J. 1997. Modulation of Tissue-Tocopherol in African Catfish, Clarias gariepinus (Burchell), Fed Oxidized Oils, and the Compensatory Effect of Supplemental Dietary Vitamin E. Aquaculture Nutrition, 3: 91-97.

Birk, Y. and Peri, I. 1980. Saponins. (In: Toxic Constituents of Plant Food Stuffs. Ed. Liener I. E.), Academic Press, New York, 161-182.

Burits, M. and Bucar, F. 2000. Antioxidant Activity of Nigella sativa Essential Oil. Phytotherapy Research, 14: 323-328.

Cheikh-Rouhou, S., Besbes, S., Hentati, B., Blecker, C., Deroanne, C. and Attia, H. 2007. Nigella sativa L.: Chemical Composition and Physicochemical Characteristics of Lipid Fraction. Food Chemistry, 101: 673-681.

El-Dakhakhny, M. 1965. Studies on the Egyptian Nigella sativa L: IV. Some Pharmacological Properties of the Seeds' Active Principle in Comparison to its Dihydro Compound and its Polymer. Arzneimittelforschung, 15: 1227-9.

El-Dakhakhny, M., Mady, N. J., Lemebert, N. and Ammon, H. P. 2002. Nigella sativa Oil, Nigellone and Derived Thymoquinone Inhibit Synthesis of 5-Lipoxygenase Products in Poly Morphonuclear Leukocytes from Rats. Journal of Ethno pharmacology, 81: 161-4.

El-Emary, N. A. 1993. Egyptian Medicinal Plants: An Overview. Assiut Journal Environmental Studies, 2: 18-19.

El-Fatatry, H. M. 1975. Isolation and Structure Assignment of an Antimicrobial Principle from the Volatile Oil of Nigella sativa L. Seeds. Pharmazie, 30: 109-111.

El-Tahir, K. E. H. and Bakeet, D. M. 2006. The Black Seed Nigella sativa Linnaeus - A Mine for Multi Cures: A Plea for Urgent Clinical Evaluation of its Volatile Oil. Journal of Taibah University Medicine Science, 1: 1-19.

Gad, A. M., El-Dakhakhny, M. and Hassan, M. 1963. Studies on the Chemical Constitution of Egyptian Nigella sativa L. Oil. Planta Medica, 11: $134-138$.

Goddard, S. 1996. Feed Management in Intensive Aquaculture. Chapman and Hall, New York, 194p.

Goreja, W. G. 2003. Black Seed: Nature's Miracle Remedy. New York, Amazing Herbs Press. 55p

Greenish, H. G. 1880. Contribution to the Chemistry of Nigella sativa. Pharmaceutical Journal and Transactions, 10: 909-915.

Hanafy, M. S. M. 1989. Effect of Chemical Fertilization and Salinity on Production of Herb and Essential Oil of Marjoram Plants (Marjoram hortensis Moench). PhD. thesis, Faculty of Agriculture at Cairo University, Egypt. 
Huang, C. H. and Huang, S. L. 2004. Effect of Dietary Vitamin E on Growth, Tissue Lipid Peroxidation, and Liver Glutathione Level of Juvenile Hybrid Tilapia, Oreochromis Niloticus $\times$ O. Aureus, Fed Oxidized Oil. Aquaculture, 237: 381-389.

Hung, S. S. O., Storebakken, T., Cui, Y., Tian, L. and Einen, O. 1997. High Energy Diets for White Sturgeon Acipenser Transmontanus Richardson. Aquaculture Nutrition, 3: 281-286.

Hutchinson, J. 1959. The Families of Flowering Plants. Oxford The Charendon Press, V:1, P: 21-27. In African Catfish, Clarias gariepinus (Burchell), Fed Oxidized Oils, and The Compensatory Effect of Supplemental Dietary Vitamin E. Aquaculture Nutrition, 3: 91-97.

Ikedo, S., Shimoyanadaand, M. and Watanabe, K. 1996. Interaction Between Bovine Serum Albumin and Saponin as Studied by Heat Stability and Protease Digestion. Journal Agriculture Food Chemistry, 44: 792-795.

Jauncey, K. and Ross, B. 1982. A Guide to Tilapia Feeds and Feedings. Institute of Aquaculture, University of Stirling, Scotland, $111 \mathrm{pp}$.

Kestemont, P., Vandeloise, E., Me'lard, C., Fontaine, P. and Brown, P. B. 2001. Growth and Nutritional Status of Eurasian Perch (Perca Fluiatilis) Fed Graded Levels of Dietary Lipids With or Without Added Ethoxyquin. Aquaculture, 203: 85-99.

Koshio, S., Ackman, R. G. and Lall, S. P. 1994. Effects of Oxidized Herring and Canola Oils in Diets on Growth, Survival, and Flavor of Atlantic Salmon, Salmo salar. Journal of Agricultural and Food Chemistry, 42: 1164-1169.

Lovell, R. M. 1980. The Chemical Biology of Fishes. Academic Press, London, 943p.

Mahmoud, M., El-Abhar, H. S. and Saleh, S. 2002. The Effect of Nigella sativa Oil Against the Liver Damage Induced by Schistosoma mansoni infection in mice. Journal of Ethnopharmacology, 79: 1-11.

Malik, M. E. K. and Mohsen, M. K. 2006. Influence of Some Medicinal Plants Supplementation as a Feed Additives on Growth and Feed Utilization inDiets of Nile Tilapia (Oreochromis niloticus) Fingerlinges, Egypt. Journal of Aquatic Biologyand Fish. 11: 213-229.

Martins, D. A., Afonso, L. O. B., Hosoya, S., Lewis-McCrea, L. M., Valente, L. M. P. and Lall, S. P. 2007. Effects of Moderately Oxidized Dietary Lipid and the Role of Vitamin $E$ on the Stress Response in Atlantic Halibut (Hippoglossus Hippoglossus L.). Aquaculture, 272: 573-580.

Meral, I., Yener, Z., Kahraman, T. and Mert, N. 2001. Effect of Nigella sativa on Glucose Concentration, Lipid Peroxidation, Anti-Oxidant Defence System and Liver Damage in Experimentally Induced Diabetic Rabbits. Journal of Veterinary Medicine. A, Physiology, Pathology, Clinical Medicine, 48: 593-599.

Moltschaniwskyj, N. A. and Martinez, P. 1998. Effect of Temperature and Food Levels on the Growth and Condition of Juvenile Sepia elliptica (Hoyle 1885): an Experimental Approach. Journal of Experimental Marine Biology and Ecology, 229: 289-302. 
Nickavar, B., Mojab, F., Javidnia, K. and Roodgar, M. A. 2003. Chemical Composition of the Fixed and Volatile Oils of Nigella sativa L. Iran. Zeitschrift. Fur. Naturforschung, 58: 629-631.

Potter, S., Flores, R., Pollack, J., Loneand, T. and Jimenez, M. 1993. ProteinSaponin Interaction and its Influence on Blood Lipids. Journal Agriculture Food Chemistry, 41: 1287-1291.

Pradeep, K. U. P., Geeranl, B. O., Eggum and Uma. 1991. Influences of Spices on Utilization of Sorghum and Chickpea Protein. Plant Foods for Human Nutrition, 41: 269-279.

Rathee, P. S., Mishra, S. H. and Kaushal, R. 1982. Antimicrobial Activity of Essential Oil, Fixed Oil and Unsaponifiable Matter of (Nigella sativa L.). Indian Journal of Pharmacology Science, 44: 8-10.

Rossell, J. B. 1986. Classical Analysis of Oils and Fats. (In: Analysis of Oils and fats. Ed. R. J. Hamilton \& J. B. Rossell). England: Elsevier Applied Science, 1-32.

SAS. 2000. Statistical Analysis Systems, SAS Users Guide, Statistics. SAS Institute, Gary North Carolina.

Satoh, S., Takeuchi, T. and Watanabe, T. 1984. Effects of Starvation and Environmental Temperature on Proximate and Fatty Acid Compositions of Tilapia Niloticus. Bulletin of the Japanese Society of Scientific Fisheries, 50: 79-84.

Schrägle, R. and Müller, W. 1990. The Influence of Selected Tannin Containing Plants Species on the Tenacity of Pathogenic Bacteria in vitro Rumen System. 1. Communication: The Influences of Selected Plants Species on the Tenacity of $\mathrm{Cl}$. Perfringens. Zentralb Veterinarmed. 2nd Cong. Faculty of Veterinary Medicine, Cairo University, 37: 30-181

Soliman, A. K., Atwa, A. M. R. and Abaza, M. A. 2000. Partial Replacement of Fish Meal Protein with Black Seed Meal Protein, with and without Lysine and Methionine Supplementation, in Diets of Nile Tilapia (Oreochromis niloticus). The Fifth International Symposium on Tilapia Aquaculture, Conf. Proc. Rio De Janeiro- RJ, Brazil, 7: 187 - 196.

Steffens,W.1989.Principles of fish nutrition, John Wiley\&Sons,New York, $108 p$.

Steel, R. G., Torrie, J. H. and Dickey, D. A. 1997. Principles and Procedures of Statistics: A Biometrical Approach. Mcgraw-Hill Co. 666p.

Swamy, S. M. K. and Tan, B. K. H. 2001. Extraction, Isolation and Characterization of Antitumor Principle, a-hederin from the seeds of Nigella sativa, Planta Medica, 67: 29-32.

Tacon, A. G. J. 1990. Standard Methods for the Nutrition and Feeding of Farmed Fish and Shrimp. Argent Laboratories Press, Washington. 295p.

\section{RESPONSE OF NILE TILAPIA OREOCHROMIS NILOTICUS TO FED DIETS SUPPLEMENTED WITH GRADED LEVELS OF BLACK SEED NIGELLA SATIVA L. STORED UNDER TROPICAL CONDITIONS Khaled A. Qatnan and Mohammed A. Al-Owafeir*}




\title{
* Dept. of Animal and Fish Production, College of Agricultural and Food Sciences, King Faisal University,
} PO Box 55135, Hofuf, 31982, Saudi Arabia, malowafeir@kfu.edu.sa

\begin{abstract}
A trial was conducted on Nile tilapia Oreochromis niloticus with intial weight $0.83 \pm 0.04 \mathrm{~g} / \mathrm{fish}$, for 12 weeks in aquatic lab of department of Animal and Fish Production in the College of Agricultural and Food Sciences, to investigate the effects of black seed Nigella sativa on feed intake, growth, and general performance. Five balanced diets were prepared (equal in protein and energy content) at various concentrations of black seed $(0,0,5,10$ and $20 \mathrm{~g} / \mathrm{kg})$, and stored at similar conditions that corresponding to prevailing enviromental conditions of kingdom of Saudi Arabia fish farms, particularly those exposed to temperature, Humidity and direct sunlight for 4 months. However, dueuar suwk of $(0 \mathrm{~g})$ was preserved at $4 \mathrm{C}^{\circ}$ as standard until used. Feeding of fish was three times a day until satiation. The results of peroxide analysis of diets demonstrated that the oxidation of feed components (free of black seed) which stored in prevailing conditions of Saudi ongoing fish farms exceeded the acceptable level in feed with amount of $17.63 \mathrm{ml}$ equivalent $/ \mathrm{kg}$, while other feeds were remained within acceptable levels. On the other hand, results of the study showed significant differences between control treatment and other treatments, in term of final weight, gained weight, Specific growth rate, consumed feed, feed conversion ratio, protein efficiency yatioand protein productive value. Regarding the survival rate, the results showed that no significant differences between the control treatment fish and other treatments except with second treatmen (free of black seed) which survival rate was reduced to $30 \%$. Results affirmed no significant differences between control treatment and other treatments which contained black seed at concentrations $5 \mathrm{~g}, 10 \mathrm{~g}$ and $20 \mathrm{~g} 1 \mathrm{~kg}$ diet, in term of crude protein and ether extract; while there were significant differences between control treatment and second treatment (free black seed), and between the second treatment and other treatments in crude protein and ether extract. In addition, there was a significant difference in the content of fish moisture between control treatmen and other treatments. Also, there were no significant differences between control treatment and other treatments in ash content. Keywords:Black seed, tilapia, tila, oriochromis nilotics fish diets,
\end{abstract}

